\title{
Verzeichnis der Gesellschaften
}

Aachener und Münchener Lebensversicherungs-Aktiengesellschaft, Karlsruhe i. B., Ritterstraße 3, Ruf 28612, Drahtwort: Lebenschutz, Geschäftsstelle Groß-Berlin: Berlin-Schöneberg, Badensche Straße 2, Ruf 714753 . . . . . . . . .

Agrippina Lebensversicherungs-Aktiengesellschaft, Berlin W 30 , Mackensenstr. 16, Ruf 2416 65/67, Drahtwort: Agrippinaleben, Zweigniederlassung: Köln, Riehler Str. 90, Ruf 73723 u. 74786

Albingia Lebensversicherungs-Aktiengesellschaft, Hamburg 1, Ballindamm 39, Ruf 3210 02, Drahtwort: Albingia, Fernschreiber 021 1774. Bezirksdirektion Berlin, Berlin-Schöneberg, Innsbrucker Str. 26/27, Ruf 716295. . . . . . . . . . . . .

Allgemeine Assekuranz (Assicurazioni Generali), Triest. Hauptbevollmächtigter für Deutschland: Carl Rieck, Hamburg 1, Mönckebergstraße 31, Ruf 330355/56, Drahtwort: Versrieck, Fernschreiber 0212880 , Berlin-Charlottenburg, Kaiserdamm 32, Ruf 924781 u. 923218 . . . . . . . . . . . . . . .

Allgemeine Rentenanstalt Lebens- und Rentenversicherungs-AG., Stuttgart S, Tübinger Straße 22, Ruf $77351 / 53$, Drahtwort: Rentenanstait. Bezirksdirektion Berlin: Berlin-Schöneberg, Fritz-Elsaß-Str. 9/10. Ruf 715513 . . . . . . . . . . . .

Allianz Lebensversicherungs-AG., Hauptverwaltung: Stuttgart W, Silberburgstr. 174, Ruf $68141 / 68147$, Fernschreiber 0723571 . Berlin W 15, Joachimstaler Straße 10-12, Ruf 910411, Fern. schreiber 0283810 . München 22, Ludwigstraße 12, Ruf 28431 , Fernschreiber 0523653, Drahtwort: Allianzleben . . . . . .

Alte Leipziger Lebensversicherungsgesellschaft auf Gegenseitigkeit, Frankfurt a. M. .Bockenheimer Landstraße 42, Ruf 71451 u. 71959 , Drahtwort: Alteleip. Bezirksdirektion Berlin: Berlin-Schöneberg, Martin-Luther-Straße 54, Ruf 710148

Alte Volksfürsorge Gewerkschaftlich-Genossenschaftliche Lebensversicherungsaktiengesellschaft, Hamburg 1, An der Alster 57/61, Ruf 248051 , Drahtwort: alvovers, Fernschreiber 0211300 . Geschäftsstelle Berlin: Berlin-Wilmersdorf, Hohenzollerndamm 174/177, Ruf 874782 . . . . . . . . . . . . .

Atlas Lebensversicherungs-Aktiengesellschaft, Heidelberg, Treitschkestraße 3, Ruf 3293, Drahtwort: Atlas . . . . .

Basler Lebens-Versicherungs-Gesellschaft, Basel, Albananlage 7, Ruf 2218 30. Hauptverwaltung für die Bundesrepublik Deutschland: Frankfurt a.M., Mendelssohnstraße80, Ruf 738 40, Drahtwort: Baslerleben. Zweigniederlassung: Berlin W 15, Kurfürstendamm 52 (Haus Basel), Ruf 912844 . . . . . . . . . .

Bayerische Beamtenversicherungsanstalt, Allgemeiner Lebensversicherungsverein auf Gegenseitigkeit, Mühchen 2, Lenbachplatz 4, Ruf 524 41-45, Drahtwort:Beamtenleben. Bezirksdirektion Berlin: Berlin-Charlottenburg 2, Berliner Straße 153, Ruf $346052 \ldots \ldots \ldots 11$ 
Berlinische Lebensversicherung Aktiengesellschaft, Berlin SW 68, Markgrafenstraße 11, Ruf 610471, Fernschreiber 0283542, Wiesbaden, Schillerplatz 2, Ruf 59816, Drahtwort: Berlinische, Fernschreiber 0416850 . . . . . . . . . . . .

Bonner Lebensversicherung Aktiengesellschaft, Köln, Hansaring 40-46, Ruf 2256 42, Bezirksdirektion Berlin: Berlin-Charlottenburg 5, Kaiserdamm 15, Ruf 920161 . . . . . . . . . .

Braunschweigische Lebensversicherung A. G., Braunschweig, Jasperallee 86/87, Ruf $25451 / 52 / 53$, Drahtwort: Braunleben. Bezirksdirektion Berlin: Berlin-Schöneberg, Innsbrucker Straße 28, Ruf 711365 . . . . . . . . . . . . . . .

Concordia Lebensversicherungs-Aktiengesellschaft, Köln, MariaAblaß-Platz 1, Ruf 29 01, Drahtwort: Concordialeben, Fernschreiber 088666 , Verwaltung Großberlin: Berlin W 15, Kurfürstendamm 52, Ruf $914553 / 54$. . . . . . . . . . .

Debeka Sterbegeld- und Lebensversicherungsverein auf Gegenseitigkeit, Koblenz, Süd-Allee 15-19, Ruf 2375, Drahtwort: Debeka, Geschäftsstelle Berlin: Berlin-Wilmersdorf, Berliner Straße 36, Ruf $872472 / 73$. . . . . . . . . . . . . . .

Der Anker Allgemeine Versicherungs-Aktiengesellschaft, Wien I, Hoher Markt 12, Direktion für Deutschland: Frankfurt a. M., Kaiserstr. 72, Ruf 32140, Filialdirektion Berlin: Berlin W 15, Meinekestraße 5, Ruf 912779 . . . . . . . . . . . .

Deutsche Anwalt- und Notar-Versicherung, siehe unter Hansa Lebensversicherung a. G. . . . . . . . . . . . 34a

Deutsche Ärzteversicherung auf Gegenseitigkeit, Berlin-Zehlendorf, Potsdamer Straße 47/48, Ruf 843536/37, Hamburg 1, Danziger Straße 35a, Ruf 244047 . . . . . . . .

Deutsche Beamten-Versicherung Offentlichrechtliche Lebensund Renten-Versicherungsanstalt, Berlin W 15, Knesebeckstraße 59/60, Ruf $914144 / 45 / 46$, Drahtwort: Beamtenleben

Deutsche Eisenbahn-Versicherungskasse Lebensversicherungsverein a. G., Sitz Berlin, Hauptleitung Köln, An der Münze 12-18, Dienstfernruf 2285/1 1, Postfernruf 72858, Bezirksleitung Berlin: Berlin W 35, Kluckstraße 25, Ruf 244788 . . . . .

Deutscher Herold Volks- und Lebensversicherungs-AG., Bonn, Poppelsdorfer Allee 31/33, Ruf 31851, Fernschreiber 089653, Drahtwort: Heroldleben, Berlin SW 48, Friedrichstraße 219/220,

Deutscher Lloyd Lebensversicherung Aktiengesellschaft, München 15, Bayerstraße 47, Ruf 57021 , Drahtwort: Lloydleben. Geschäftsstelle Berlin: Berlin-Friedenau, Bornstraße 1, Ecke Bundesallee (Lloydhaus), Ruf 839561

Deutscher Ring Lebensversicherungs-Aktiengesellschaft, Hamburg 36, Karl-Muck-Platz 1, Ruf 3414 41, Drahtwort: Ringsicherung. Filialdirektion Berlin: Berlin W 30, Tauentzienstraße 18a, Ruf $242551,242552,241767$. . . . . . .

Eos Volks- und Lebensversicherungs-Aktiengesellschaft, Düsseldorf, Hauptverwaltung Coburg bis 31. XII. 1955, Herrngasse 11, Ruf 35 49, Drahtwort: Eos. Bezirksdirektion Berlin: Berlin W 30 , Geisbergstraße 11, Ruf 245253

Frieden Deutscher Bestattungs- und Versicherungsverein a. G., Berlin-Wilmersdorf, Berliner Straße 154, Ruf 878800 . . . . 
Friedrich Wilhelm Magdeburger Lebensversicherungs-Aktiengesellschaft, Köln, Enggasse, Ruf 2861, Drahtwort: Friedhelmleben, Fernschreiber 088587, Berlin-Wilmersdorf, Hohenzollerndamm 196, Ruf 870346

Gerling-Konzern Lebensversicherungs-Aktiengesellschaft, Köln, Gerling-Hochhaus, Ruf 2861, Drahtwort: Gerlingleben, Fernschreiber 08 8587, Zweigniederlassung Berlin: Berlin-Wilmersdorf, Hohenzollerndamm 196, Ruf 870346

Gilde Deutsche Versicherungs-Aktiengesellschaft, Düsseldorf, Graf-Recke-Straße 82, Ruf 66101, Bezirksdirektion Berlin: Berlin W 15, Kurfürstendamm 184, Ruf 913771, 915261 . .

Gisela Allgemeine Lebens- und Aussteuer-Versicherungs-Aktiengesellschaft, München 8, Prinzregentenplatz 7/9, Ruf 449061, Drahtwort: Giselaversicherung, Filialdirektion Berlin: BerlinWilmersdorf, Rudolstädter Straße 121, Ruf 874767 . . . .

Gladbacher Lebensversicherung Aktien-Gesellschaft, MönchenGladbach, Wallstraße 30/32, Ruf 2 45 51, Drahtwort: Gladbachleben, Bezirksdirektion Berlin: Berlin-Lichterfelde-W, EnzianstraBe 2, Ruf 760626 . . . . . . . . . . . . . . . .

Gothaer Lebensversicherung auf Gegenseitigkeit, Göttingen, Gothaer Platz 8, Ruf 3871, Drahtwort: Gothaleben, Fernschreiber 096876, Hauptbevollmächtigter für Berlin: BerlinHalensee, Kurfürstendamm 103/104, Ruf 975050. . . . .

Hamburg-Mannheimer Versicherungs-Aktien-Gesellschaft, Hamburg 36, Alsterufer 1, Ruf 4411 01, Drahtwort: Hamavag, Filialdirektion Berlin: Berlin W 15, Meinekestraße 3, Ruf 912850, 917850 .

Hannoversche Lebensversicherung auf Gegenseitigkeit zu Hannover, vormals Preußischer Beamten-Verein, Raschplatz 13, Ruf 242 46, Drahtwort: Hannoverleben, Büro Berlin W 15. Kurfürstendamm 224, Ruf 914859 . . . . . . . . . .

Hansa Lebensversicherung a. G., Hamburg 36, Alsterufer 1, Ruf 442241, Drahtwort: Hansaleben, Filialdirektion Berlin: Berlin W 15, Meinekestraße 3, Ruf 912850 . . . . . . . .

Hansa (Sonderabteilung: Deutsche Anwalt- und Notar-Versicherung) Hamburg 1, Mönckebergstraße 11, Ruf 338958, Drahtwort: Anwaltpension .............. 34a

Iduna-Germania Lebens-Versicherungsgesellschaft auf Gegenseitigkeit in Berlin, siehe unter Vereinigte Lebensversicherungsanstalt a. G. für Handwerk, Handel und Gewerbe, Hamburg

Isar Lebensversicherungs-Aktiengesellschaft, München 23, Giselastraße 21/23, Ruf 39221, Drahtwort: Isarag, Zweigstelle Berlin: Berlin W 30 , Geisbergstraße 11, Ruf 244272 .. . . . .

Karlsruher Lebensversicherung A.-G., Karlsruhe i. B., FriedrichScholl-Platz, Ruf 25974, Drahtwort: Karlsruherleben, Fernschreiber 0782846, Bezirksdirektion Groß-Berlin: Berlin W 30, Hardenbergstraße 15, Ruf 322282 . . . . . . . . . . . .

Kölnische Lebensversicherung a. G., Köln, Clever Straße 36-38, Ruf 74948, Fernschreiber. 088366, Bezirksdirektion Berlin: Berlin SW 61, Tempelhofer Ufer 14, Ruf 665511. . . . . .

Lebensversicherungsanstalt Berlin, Körperschaft des öffentlichen Rechts, siehe unter öffentlich-rechtliche Lebensversicherungsanstalten . . . . . . . . . . . . . . 63b 
Leipziger Verein-Barmenia Lebensversicherung auf Gegenseitigkeit, Hamburg-Volksdorf, Halenreie 40-44, Ruf 624441, Drahtwort: Elvaubeleben, Bezirksdirektion Berlin: Berlin-Charlottenburg, Bismarckstraße 45, Ruf $346014 / 15$. . . . . . . . .

Magdeburger Allgemeine Lebens- und Rentenversicherungs-Aktiengesellschaft, Fulda, Heinrich-von-Bibra-Platz, Ruf 3561, Drahtwort: Allgema, Niederlassung Berlin: Berlin W 30, Bamberger Straße 44, Ruf 244019

Magdeburger Lebens-Versicherungs-Gesellschaft, siehe unter Friedrich Wilhelm Magdeburger Lebensversicherungs-Aktiengesellschaft, Köln

Mannheimer Lebensversicherungs-Gesellschaft A.-G., Berlin W 30, Rankestraße 23, Ruf 242414/15, Mannheim, E 5, Technisches Rathaus, Ruf $31613 / 15$

Münchener Lebensversicherungsanstalt $\mathrm{AG}$, München 23, Leopoldstraße 6, Ruf 38945-47, Drahtwort: Münchenerleben, Fernschreiber 0523192

Münchener Verein Lebens- und Altersversicherungsanstalt a. G., München 3, Pettenkoferstraße 19, Ruf 50441 . . . . . . .

National Lebensversicherungs-Aktien-Gesellschaft, Lübeck, Travemünder Allee 9, Ruf 25321 , Drahtwort: National, Fernschreiber 02 6850, Direktion für Groß-Berlin: Berlin SW 68, Friedrichstraße 31, Ruf 617627 . . . . . . . . .

Nord-Deutsche Lebensversicherungs-Aktiengesellschaft, Hamburg 11, Alterwall 12, Ruf $341301 / 06$, Drahtwort: Nordleben, Hauptverwaltungsstelle Berlin: Berlin-Charlottenburg, Württembergallee 1, Ruf 940736 . . . . . . . . . .

Nordstern Lebensversicherungs-Aktiengesellschaft, Köln, KaiserFriedrich-Ufer 23, Ruf 70831, Fernschreiber 088731. BerlinSchöneberg, Nordsternplatz, Ruf 710331, Fernschreiber 0283511 , Drahtwort: Nordsternleben . . . . . . . .

Nürnberger Lebensversicherung AG, Nürnberg, Rathenauplatz 16-18, Ruf 514 41, Drahtwort: Lebensbank, Fernschreiber 062163. Direktionsgeschäftsstelle Berlin: Berlin-Charlottenburg 9, Reichskanzlerplatz 4, Ruf 924997 . . . . . . . 46

Oeffentlich-rechtliche Lebensversicherungsanstalten ... . . 63b Verzeichnis der einzelnen Anstalten siehe Seite $63 \mathrm{~b}$

Pensionsverein Deutscher Pensionszuschuß- und Lebensversicherungsverein a. G., München 15, Beethovenplatz 4, Ruf $52741 / 42$

Prima Lebensversicherungsverein a. G., Nürnberg 2, Frauentorgraben 11/13, Ruf 27554 , Drahtwort: Prima . . . . . . .

Raiffeisendienst Lebensversicherungs-Gesellschaft a. G., BerlinCharlottenburg 2, Schillerstraße 3, Ruf 3238 11. Wiesbaden, Sonnenberger Straße 2, Ruf 59331, Fernschreiber 0416803. .

Rheinisch-Westfälische Lebensversicherung a. G., WuppertalBarmen, Carnaper Straße 73-75, Ruf 566 67/8, Direktionsgeschäftsstelle Berlin: Berlin-Friedenau, Ringstraße 61/62, Ruf 836694 . . . . . . . . . . . . . . . . . .

Rothenburger Lebensversicherungs-AG. Köln siehe Concordia Lebensversicherungs-Aktiengesellschaft Köln .. . . . . . .

Schweizerische Lebensversicherungs- und Rentenanstalt, Zürich, Alpenquai 40. Niederlassung für Deutschland: München 23, Leopoldstraße 8, Ruf $34251 / 53$, Drahtwort: Schweizerische München . . . . . . . . . . . . . . . . 
Sparkassen-Versicherung AG., Stuttgart-N, Friedrichstraße 4, Ruf 95646 .... . . . . . . . . . . . . .

Universa Lebensversicherungsanstalt auf Gegenseitigkeit, Nürnberg, Sulzbacher Straße 1-5, Ruf 53451/53 . . . . . . . . 53

Verband offentlicher Lebensversicherungsanstalten in Deutschland, Düsseldorf, . . . . . . . . . . . . . 63b

Vereinigte Lebensversicherungsanstalt a. G. für Handwerk, Handel und Gewerbe, Hamburg 36, Alsterufer 50, Ruf 441141 , Drahtwort: Vereinigteleben, Fernschreiber 0211397, ZweigniederIassung Iduna-Germania, Berlin SW 68, Charlottenstraße 13, Ruf 610236 , Fernschreiber 0283806. . . . . . . . . .

Victoria zu Beriin Allgemeine Versicherungs-Actien-Gesellschaft, Berlin SW 68, Lindenstraße 20-25, Ruf 610101 , Drahtwort: Victoriabank, Fernschreiber 0283477, Hauptverwaltung für den Westen: Düsseldorf, Bahnstraße 2-8, Ecke Königsallee, Ruf 8711 , Fernschreiber 0822984. . . . . . . . . . . .

Victoria am Rhein Allgemeine Versicherungs-Actien-Gesellschaft, Düsseldorf, Bahnstraße 2-8, Ecke Königsallee, Ruf 87 11, Drahtwort: Victoriarhein, Fernschreiber 0822984 . . . . . . .

Vita Lebensversicherungs-Aktiengesellschaft, Zürich, Mythenquai 10, Ruf (051) 274810 . Direktion für Deutschland: Frankfurt a. M., Kaiserstraße 3-5, Ruf 90601/90606. Zweigniederlassung Berlin: Berlin-Charlottenburg, Leibnizstraße 63, Ruf 320697 ...............

Vohk Lebensversicherungsanstalt ostdeutscher Handwerkskammern, V. a. G., zu Berlin, Berlin W 35, Potsdamer Straße 76, Ruf 246734/35, Zweigniederlassung: Hamburg 13, Johnsallee 53-55, Ruf 448566 .. . . . . . . . . . . .

Volkshilfe Lebensversicherungs-Aktiengesellschaft, Berlin-Friedenau, Sarrazinstraße 11-15, Ruf 8320 55, Fernschreiber 0283588, Köln, Kaiser-Wilhelm-Ring 46, Ruf $211641 / 43$, Fernschreiber 088862 . . . . . . . . . . . . . .

Volkswohl-Bund Lebensversicherung a. G., Berlin-Charlottenburg, Kantstraße 13, Ruf 324421, Hauptverwaltung West: Dortmund, Südwall 37-39, Ruf 22441/43 . . . . . . . . . .

Winterthur Lebensversicherungs-Gesellschaft in Winterthur (Schweiz), Direktion für die Bundesrepublik Deutschland: München 23, Leopoldstraße 34-36, Ruf 360721 , Fernschreiber 0523145 . . . . . . . . . . . . . . . . . . . .

Witwen- und Waisenkasse des Reichs- und Staatsdienstpersonals Allgemeine Lebensversicherungsanstalt a. G., München 2, Marsstraße 22-23a, Ruf 58491 . . . . . . . . . . .

Württembergischer Versicherungsverein a. G. Stuttgart, Stuttgart S, Mörikestraße 5, Ruf 76236/38, Drahtwort: Württleben

Verband der Lebensversicherungsunternehmen e. V.

Bonn, Bonner Talweg 33, Ruf $33751 / 52$,

Drahtwort: Lebensverband.

Verbandsdirektor: Dr. Waldemar Adler. 
